\title{
DERIVING A SIMULATION MODEL OF A 40-KHZ CMUT CELL FROM IMPEDANCE AND INTERFEROMETER MEASUREMENTS
}

\author{
I.O. Wygant ${ }^{1,2^{*}}$, M. Kupnik ${ }^{l}$, and B.T. Khuri-Yakub ${ }^{l}$ \\ ${ }^{1}$ Stanford University, Stanford, California, USA \\ ${ }^{2}$ National Semiconductor, Santa Clara, CA, USA
}

\begin{abstract}
A spring-mass-damper system and corresponding equivalent circuit help design and analyze capacitive micromachined ultrasonic transducers (CMUTs). The ability to extract similar models from measured devices helps design verification and development of simulation models that match fabricated devices. Impedance measurements yield the CMUT's clamped capacitance and motional impedance, which fits a 4-element equivalent circuit. Additional measurements help extract a more complete equivalent circuit that includes the electromechanical transformer ratio and effective mechanical elements. In this work, we combine impedance measurements, the tightly specified mass of the CMUT plate, and interferometer displacement measurements to compute a more complete equivalent circuit. The circuit obtained for two 40$\mathrm{kHz}$ CMUT cells exhibits a good fit to the model derived from the device geometry alone. The experimentally-obtained model exhibits larger damping which is likely due to energy loss in addition to radiated ultrasound.
\end{abstract}

\section{INTRODUCTION}

Capacitive micromachined ultrasonic transducers (CMUTs) are ultrasonic transducers fabricated using microfabrication techniques. CMUTs find use in a variety of applications including airborne ultrasound, medical imaging, medical therapy, and chemical sensing.

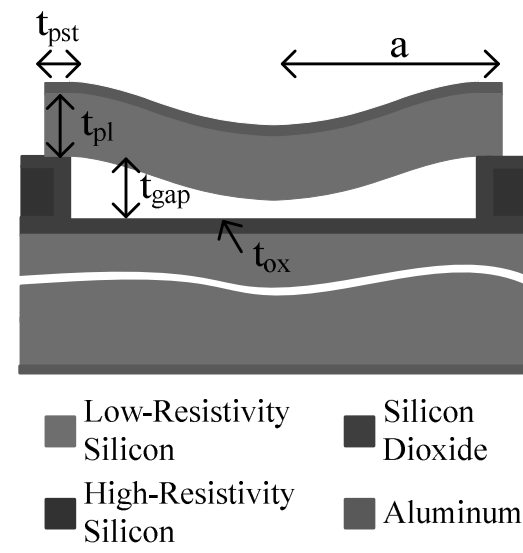

(a)

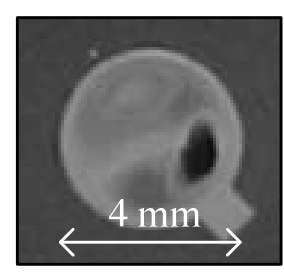

(b)
Figure 1: A single 40-kHz CMUT cell. (a) Diagram of cell cross section. (b) Photograph of a single cell that was mounted on a printed circuit board for testing.

Development of CMUTs for a particular application benefits from careful characterization of fabricated devices. This characterization helps evaluate the accuracy of models used to design the devices, and, furthermore, helps develop models for interface circuit design and signal processing that match measured performance.

Impedance measurements are particularly useful for characterizing fabricated CMUTs. Impedance is conveniently measured at wafer level without support circuitry and provides device information over a wide frequency range.

Early work on capacitive ultrasonic transducers made extensive use of impedance measurements and equivalent circuits. Simple inspection of impedance graphs yields information about resonance frequency, quality factor, and efficiency [1]. Linking the measured impedance with equivalent circuits of mechanical structures aids design and analysis [2]. Continuing advances in CMUT analysis [3], equivalent circuit modeling, and simulation [4] enables extraction of even more utility from impedance measurements.

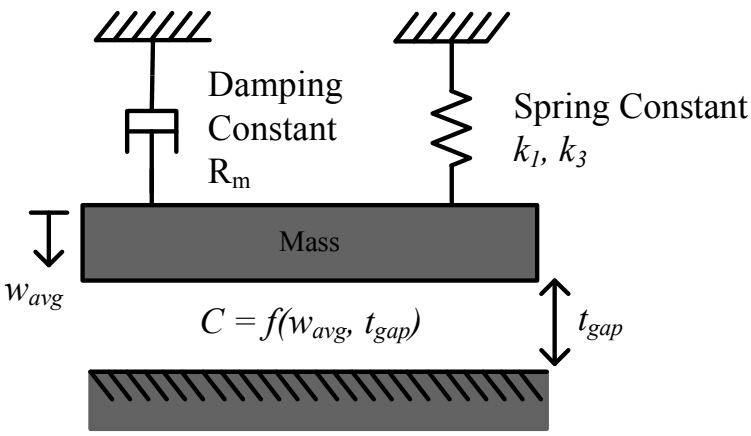

Figure 2: A spring-mass-damper model of the CMUT.

A single impedance measurement gives the impedance of the clamped capacitance in parallel with the motional impedance. From this data we can extract a basic 4-element model of the device. For example, [5] describes extracting the effective springconstant, equivalent mass, and damping components of the motional impedance. In [6], impedance data measured as a function of bias voltage helps separate the effects of spring softening from the mechanical spring. The components of the 4element model gives information about resonance frequency, quality factor, and energy dissipation, but do not give acoustic parameters such as radiated power or source pressure. To model these, we need to extract additional circuit parameters.

Additional measurements can provide these parameters. In [5], variation in static pressure helps isolate sources of damping. Impedance measurements made in gases with different acoustic impedances gives information about the radiation impedance [1]. These techniques, however, don't give the electromechanical transformer ratio $n$, which couples the acoustic and mechanical parts of the equivalent circuit. In [1], Hunt suggests making an additional impedance measurement with a known mass on the device to extract the transformer ratio; however, this technique is not practical for many MEMS devices. In this work, we leverage interferometer measurements and the tight specification of certain device dimensions and material properties to extract an electromechanical model of a $40-\mathrm{kHz}$ CMUT air transducer.

\section{DEVICE GEOMETRY AND CLAMPED PLATE ANALYSIS}

In this work, we analyze single CMUT cells designed for generating high-intensity ultrasound for a directional audio application. We fabricated the devices using the wafer-bonding 
process described in [7]. Fig. 1 shows the basic device geometry and a picture of a typical cell. Table 1 gives the critical dimensions of the two characterized devices.

\begin{tabular}{|l|l|l|}
\hline Device & 1 & 2 \\
\hline Cell radius, $a(\mathrm{~mm})$ & 2.22 & 2.08 \\
\hline Post width, $t_{p s t}(\mu \mathrm{m})$ & 75 & 75 \\
\hline Plate thickness, $t_{p l}(\mu \mathrm{m})$ & 40 & 40 \\
\hline Oxide thickness, $\mathrm{t}_{\mathrm{ox}}(\mu \mathrm{m})$ & 5 & 5 \\
\hline Gap thickness, $\mathrm{t}_{\text {gap }}(\mu \mathrm{m})$ & 60 & 60 \\
\hline
\end{tabular}

Table 1: Device cell dimensions.
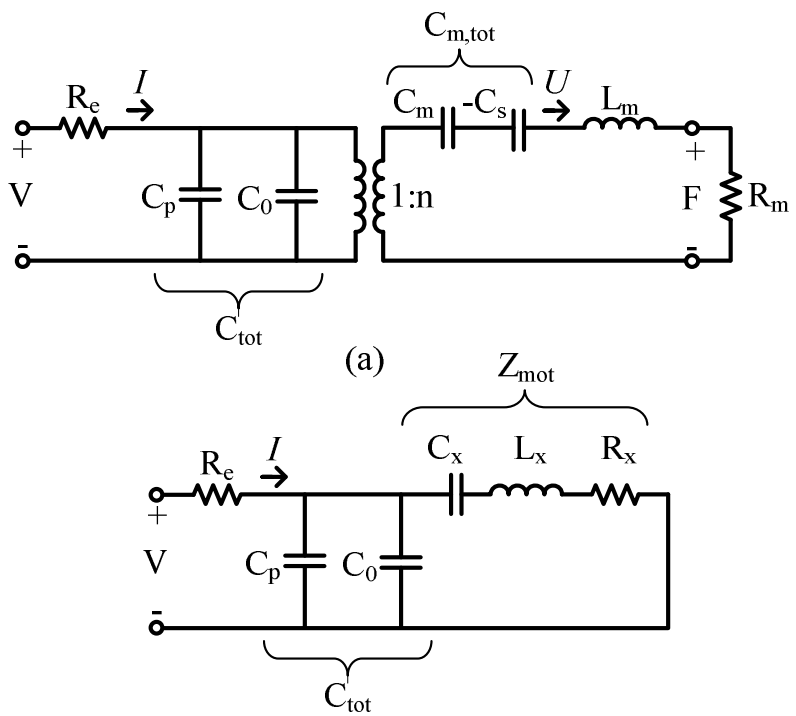

(b)

Figure 3: Small-signal CMUT equivalent circuit models. (a) Full electromechanical model. (b) Model with all components referred to the electrical side. Fitting to the measured impedance data yields values for the electrically-referred components. $R_{e}$ is an optional series resistance representing contact resistance.

Assuming a perfectly clamped plate, we can derive a springmass-damper model of the cells (Fig. 2) [3]. The effective spring constant equals (1), where $D$ is the flexural rigidity given by (2). In these expressions, $a$ is the plate radius, $t$ is the plate thickness, $E$ is the Young's modulus of silicon (148 GPa), and $v$ is the Poisson ratio for silicon (0.177).

$$
\begin{aligned}
& k_{1}=\frac{192 \pi D}{a^{2}}(1) \\
& D=\frac{E t^{3}}{12\left(1-v^{2}\right)}
\end{aligned}
$$

Because the static plate deflection of the cells is significant relative to their thickness, tensile membrane forces described by $k_{3}$ affect the deflection.

$$
k_{3}=4.1 \frac{k_{1}}{t^{2}}
$$

Finally, the effective mass of the plate equals (4), where $\rho$ equals the density of silicon $(2330 \mathrm{~kg} / \mathrm{m} 3)$.

$m=1.84 \pi a^{2} \rho t$ (4)

Damping is the most complex parameter to predict. Although we expect multiple sources of damping such as energy lost to the substrate and posts, we assume damping is due solely to radiated ultrasound. The radiation impedance of a clamped circular cell is given in [8] and approximately equals (5), where $k$ is $2 \pi$ divided by the wavelength in air and $Z_{0}$ equals the acoustic impedance of air (409 Rayls).

$$
Z_{\text {rad }} \simeq Z_{0} \frac{(k a)^{2}+j k a}{1+\left(k a^{2}\right)}(5)
$$

With these equations we compute the spring-mass-damper parameters given in the last three rows of Table 2.

The capacitance as a function of gap is given by (6). In this expression, $w_{\text {avg }}$ equals the average plate deflection, which is $1 / 3$ the peak deflection, and $g_{0}$ is the effective gap, which equals the gap height plus the oxide thickness divided by its dielectric consgtant (3.78 for $\mathrm{SiO}_{2}$ ). With (6) and the spring-mass-damper parameters, we can calculate the equivalent circuit of the CMUT cell shown. Fig. 3 shows the CMUT equivalent circuit which includes parasitic capacitance $C_{p}$, which we calculate from the device geometry.

$$
C=\frac{\pi a^{2} \varepsilon_{0} \operatorname{atanh}\left(\sqrt{\frac{3 w_{\text {wavg }}}{g_{0}}}\right)}{\sqrt{3 g_{0} w_{\text {avg }}}}
$$

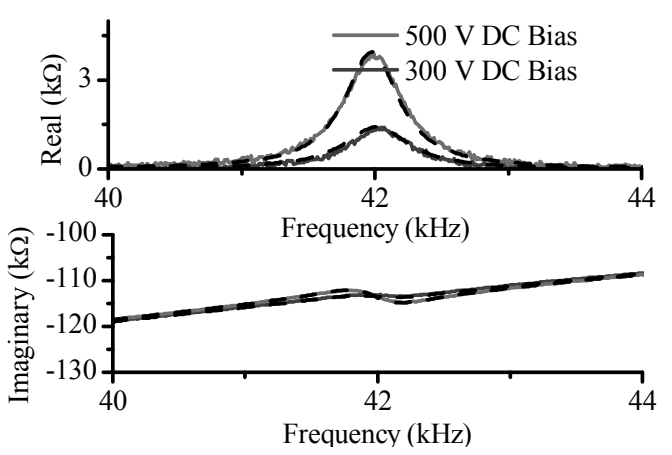

(a)

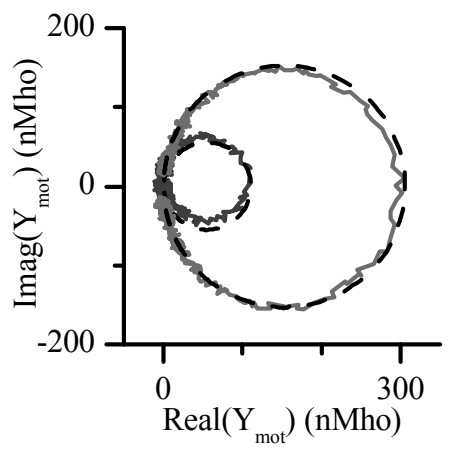

(b)

Figure 4: Electrical input impedance. (a) Real and imaginary parts of measured (solid) and fitted (dashed) input impedance. (b) Subtracting the parallel-capacitance from the impedance leaves the motional impedance [1]. A fit to the motional impedance demonstrates a simple spring-mass-damper system. 


\section{MEASUREMENTS AND ANALYSIS}

Using an impedance analyzer (Agilent 4294A, Agilent Technologies, Palo Alto, CA), we measured the input impedance of two CMUT cells mounted on printed circuit boards (PCBs). Fig. 3 shows the real and imaginary parts of the measured impedance at different bias voltages.

\begin{tabular}{|l|l|l|l|l|}
\hline & Geometry & Meas. & Geometry & Meas. \\
\hline Device & \multicolumn{2}{|c|}{1} & \multicolumn{2}{c|}{2} \\
\hline$C_{p}(\mathrm{pF})$ & 30.0 & - & 29.0 & - \\
\hline$C_{0}(\mathrm{pF})$ & 2.97 & - & 2.58 & - \\
\hline$C_{0, \text { tot }}$ & 33.0 & 33.4 & 31.6 & 32.5 \\
\hline$n(\mu \mathrm{N} / \mathrm{V})$ & 50.1 & 51.2 & 43.5 & 39.8 \\
\hline$C_{m, \text { tot }}(\mu \mathrm{m} / \mathrm{N})$ & 4.89 & 5.28 & 4.25 & 5.28 \\
\hline$L_{m}(\mu \mathrm{g})$ & 2.67 & 2.72 & 2.32 & 2.37 \\
\hline$R_{m}(\mathrm{mN} \cdot \mathrm{s} / \mathrm{m})$ & 6.63 & 8.57 & 5.77 & 10.4 \\
\hline
\end{tabular}

Table 2: Comparison of equivalent circuit parameters derived from the device geometry and from measured data for a 500-V dc bias.

From a single impedance measurement, we can extract the 4element model shown in Fig. 3(b). Key features of the impedance plot help fit a circuit to the measured data. At frequencies far from resonance, the clamped capacitance, $C_{t o t}$, dominates the imaginary part of the impedance. Thus, a least squares fit to the imaginary impedance at these frequencies yields $C_{t o t}$. Similarly, a frequencyindependent real part of the impedance indicates a series contact resistance, which for these devices was negligible.

Subtracting the contribution of $C_{t o t}$ and $R_{s}$ from the measured impedance yields the motional impedance. Plotted in Cartesian coordinates, the motional admittance should resemble a circle with additional resonances appearing as additional loops. The real part of the motional impedance equals $R_{x}$; we fit $C_{x}$ and $L_{x}$ to the imaginary part. Fig. 3 shows a visibly good fit with the equivalent circuit indicating the circuit captures the key features of the device.

To isolate the mechanical components of the equivalent circuit, we need the transformer ratio $n$, or equivalently, some mechanical value on the mechanical side of the circuit. In air, because of the high quality factor, the mass loading from the medium is negligible. Thus, we expect $L_{x}$ to equal $L_{m} / n^{2}$, where $L_{m}$ is the effective mass of the plate.

Relative to the other device parameters, the mass of the plate is well-known. Single-crystal silicon has a known density. The thickness of the plate is specified within several percent by the silicon-on-insulator (SOI) wafer manufacturer. Standard optical lithography precisely defines the plate diameter although some lateral etching of the cavities adds uncertainty about the plate diameter.

The effective mass of the plate, $L_{m}$, is a function of its mass and the mode shape. Fig. 5 shows the mode shape at the resonance frequency, which gives the effective mass according to (7).

$L_{m}=2 \pi \rho h \int_{0}^{a} w^{2}(r) r d r(7)$

The measured mode shape corresponds to an effective mass equal to 1.88 times the plate mass, which is close to the theoretical value for a clamped plate of 1.84 .

Using the known effective mass yields a value for the transformer ratio equal to (8).

$$
n=\sqrt{\frac{L_{m}}{L_{x}}}
$$

Table 2 compares the fit values with the computed values. The extracted parameters are consistent with the design. The biggest discrepancy is that the measured damping is significantly larger than predicted. However, numerous modes of energy loss exist beyond radiation impedance. Examples include energy radiated into the substrate, posts, and along the surface.
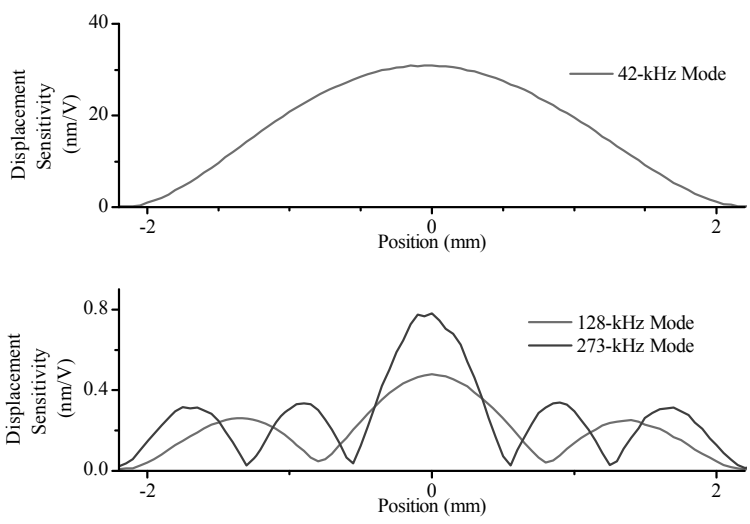

Figure 5: Cell displacement profiles and mode shapes measured with a laser vibrometer. The effective mass for the fundamental mode equals 1.88 times the mass of the cell plate, which compares to 1.84 for an ideal clamped radiator. Fig. 6 shows the impulse response and harmonics of the device, which we used to identify the mode frequencies.

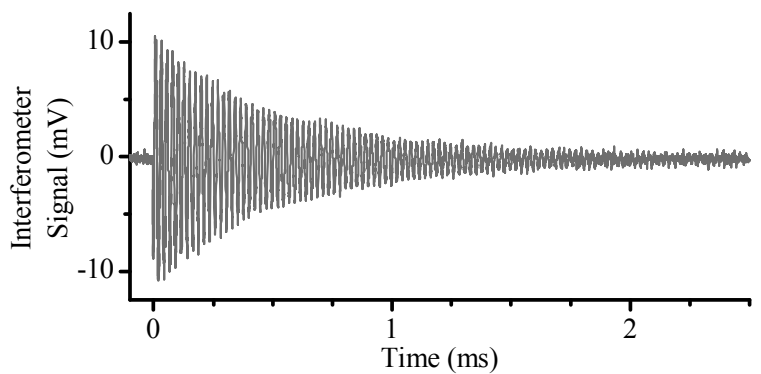

(a)

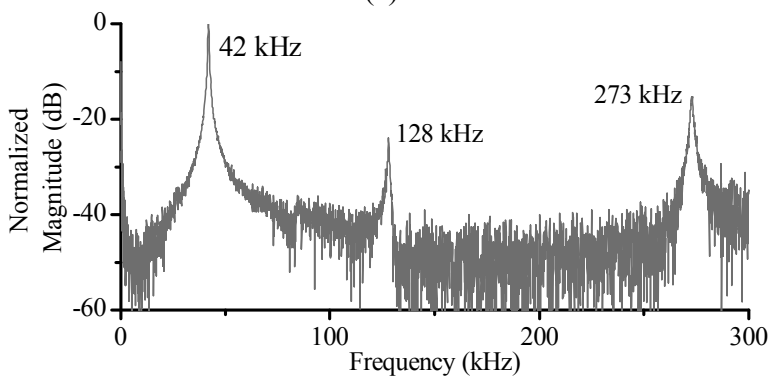

(b)

Figure 6: Impulse response measured at the device center with a laser vibrometer for a 500-V dc bias and a 20-V excitation pulse. (a) Impulse response. (b) The Fourier transform shows the fundamental and two higher-order modes. The single degree-offreedom model assumes frequencies centered about the fundamental mode. 
Although the extracted model compares well with the analytically derived model, the devices show less average displacement sensitivity than expected. Based on the measured model for device 1 given in Table 2, we expect an average displacement sensitivity of about $22 \mathrm{~nm} / \mathrm{V}$ compared to our measured average sensitivity of about $10 \mathrm{~nm} / \mathrm{V}$. Similarly, device 2 exhibits less sensitivity than the model predicts. This discrepancy in sensitivity may be due to loss mechanisms not modeled by a simple resistor and is an area for further investigation.

\section{CONCLUSION}

The characterization process described in this work yields a useful electromechanical model of the CMUT and aids comparison of design models with fabricated devices. This method leverages routine impedance measurements, the tight tolerances on certain parameters provided my microfabrication, and interferometer measurements. Pressure measured in the far field using a calibrated microphone could supplement or replace the information obtained with the interferometer.

Additional measurements could help refine the model further. Because of the significant tensile forces in the device, the effective spring constant is influenced by linear and nonlinear spring forces. Static displacement measurements would help separate the spring constants $k_{1}$ and $k_{3}$, which would be particularly useful for largesignal models.

The methodology could be extended to devices designed for operation in water, particularly since impedance testing is routinely made in air first. Additional impedance measurements and pressure measurements made with a hydrophone would help resolve the radiation impedance in water.

The process of extracting a model from measured data is important to CMUT development, and MEMS devices in general, as it closes the loop between design, test, and fabrication.

\section{REFERENCES}

[1] F. V. Hunt, Electroacoustics: The Analysis of Transduction, and Its Historical Background. Cambridge: Harvard University Press, 1982.

[2] W. P. Mason, Electromechanical Transducers and Wave Filters, 2nd ed. New York: D. Van Nostrand, 1948.

[5] I. O. Wygant, M. Kupnik, and B. T. Khuri-Yakub, "Analytically calculating membrane displacement and the equivalent circuit model of a circular CMUT cell," in IEEE Ultrasonics Symposium, 2008, pp. 2111-2114.

[6] H. K. Oguz, S. Olcum, M. N. Senlik, V. Tas, A. Atalar, and H. Koymen, "Nonlinear modeling of an immersed transmitting capacitive micromachined ultrasonic transducer for harmonic balance analysis," IEEE Transactions on Ultrasonics, Ferroelectrics and Frequency Control, vol. 57, pp. 438-447.

[3] I. J. Oppenheim, A. Jain, and D. W. Greve, "Electrical characterization of coupled and uncoupled MEMS ultrasonic transducers," IEEE Transactions on Ultrasonics, Ferroelectrics and Frequency Control, vol. 50, pp. 297-304, 2003.

[4] S. Kalicinski, H. A. C. Tilmans, M. Wevers, and I. De Wolf, "A new characterization method for electrostatically actuated resonant MEMS: Determination of the mechanical resonance frequency, quality factor and dielectric charging," Sensors and Actuators A: Physical, vol. 154, pp. 304-315, 2009.

[7] I. O. Wygant, M. Kupnik, B. T. Khuri-Yakub, M. S. Wochner, W. M. Wright, and M. F. Hamilton, "The design and characterization of capacitive micromachined ultrasonic transducers (CMUTs) for generating high-intensity ultrasound for transmission of directional audio," in IEEE Ultrasonics Symposium, 2008, pp. 2100-2102.

[8] M. Greenspan, "Piston radiator: Some extensions of the theory," The Journal of the Acoustical Society of America, vol. 65 , pp. 608-621, 1979.

\section{CONTACT}

*I.O. Wygant, tel: +1-650-566-8980; ira.wygant@nsc.com 